\title{
Bounds on the $k$-dimension of products of special posets
}

\author{
Michael Baym* Douglas B. West ${ }^{\dagger}$
}

\begin{abstract}
Trotter conjectured that $\operatorname{dim} P \times Q \geq \operatorname{dim} P+\operatorname{dim} Q-2$ for all posets $P$ and $Q$. To shed light on this, we study the $k$-dimension of products of finite orders. For $k \in o(\ln \ln n)$, the value $2 \operatorname{dim}_{k}(P)-\operatorname{dim}_{k}(P \times P)$ is unbounded when $P$ is an $n$-element antichain, and $2 \operatorname{dim}_{2}(m P)-\operatorname{dim}_{2}(m P \times m P)$ is unbounded when $P$ is a fixed poset with unique maximum and minimum. For products of the "standard" orders $S_{m}$ and $S_{n}$ of dimensions $m$ and $n, \operatorname{dim}_{k}\left(S_{m} \times S_{n}\right)=m+n-\min \{2, k-2\}$. For higher-order products of "standard" orders, $\operatorname{dim}_{2}\left(\prod_{i=1}^{t} S_{n_{i}}\right)=\sum n_{i}$ if each $n_{i} \geq t$.
\end{abstract}

\section{Introduction}

The dimension of a poset $P$ is a natural measure of its complexity. Written $\operatorname{dim} P$, the dimension is the least $t$ such that $P$ embeds as a subposet of $\mathbb{R}^{t}$ under the componentwise ordering. Note that $\mathbb{R}^{t}$ is the product of $t$ copies of $\mathbb{R}$, where the product $P \times Q$ of posets $P$ and $Q$ is the poset on $\{(p, q): p \in P, q \in Q\}$ given by $(p, q) \leq\left(p^{\prime}, q^{\prime}\right)$ if and only if $p \leq p^{\prime}$ in $P$ and $q \leq q^{\prime}$ in $Q$.

The behavior of poset dimension under the product operation is not fully understood. If $P$ embeds in $\mathbb{R}^{s}$ and $Q$ embeds in $\mathbb{R}^{t}$, then assigning $(p, q)$ the concatenation of the images of $p$ and $q$ under these embeddings shows that $P \times Q$ embeds in $\mathbb{R}^{s+t}$, and hence $\operatorname{dim}(P \times Q) \leq \operatorname{dim}(P)+\operatorname{dim}(Q)$. Since $P$ and $Q$ both appear in $P \times Q$, the trivial lower bound is $\max \{\operatorname{dim}(P), \operatorname{dim}(Q)\}$.

The upper bound is tight when $P$ and $Q$ are bounded, meaning that each has a unique maximal element and a unique minimal element. This equality was proved first by Baker [2] and later more simply by Kelly [11]. It is thought that the upper bound is never far from the truth. Trotter's conjecture that $\operatorname{dim}(P \times Q) \geq \operatorname{dim} P+\operatorname{dim} Q-2$ appears in [12].

${ }^{*}$ Mathematics Department, Massachusetts Institute of Technology, Cambridge, MA; research supported by the Fannie and John Hertz Foundation and by the National Defense Science and Engineering Program

${ }^{\dagger}$ Mathematics Department, University of Illinois, Urbana, IL 61801, west@math.uiuc.edu. Research partially supported by the National Security Agency under Awards H98230-06-1-0065 and H98230-10-1-0363. 
The fundamental result in this direction is that of Trotter [17], proving that for $n \geq 2$ the product of the smallest $n$-dimensional poset with itself has dimension $2 n-2$. Let $[n]=\{1, \ldots, n\}$. The standard example $S_{n}$ is the containment poset on the family of sets consisting of the $n$ singleton sets and their complements in $[n]$. Hiraguchi [9] showed that $S_{n}$ is the smallest $n$-dimensional poset. Reuter [15] extended Trotter's result (using "concept analysis") to show that $\operatorname{dim}\left(S_{m} \times S_{n}\right)=m+n-2$ (for $\min \{m, n\} \geq 2$ ). Reuter also showed that $\operatorname{dim}(P \times P) \geq 4$ whenever $\operatorname{dim} P=3$. Lin [13] gave another proof of Reuter's result for the product of standard examples and proved that $\operatorname{dim}\left(S_{m}^{\prime} \times S_{n}\right)=m+n-2$, where $S_{m}^{\prime}$ is the poset obtained from the standard example $S_{m}$ by adding unique maximal and minimal elements. This shows that Trotter's conjecture cannot be refined by improving the bound to $\operatorname{dim}(P \times Q) \geq \operatorname{dim} P+\operatorname{dim} Q-1$ when $P$ or $Q$ is bounded.

In this paper we extend Reuter's result on the standard examples. Let $\underline{k}$ denote the poset that is a chain of $k$ elements. The $k$-dimension of a poset $P$ is the minimum $t$ such that $P$ embeds in the product poset $\underline{k}^{t}$; the concept was introduced by Trotter [16]. Reducing $k$ restricts the flexibility of embeddings for $P$, so $\operatorname{dim}_{2} P \geq \operatorname{dim}_{3} P \geq \cdots \geq \operatorname{dim}_{\infty} P=\operatorname{dim} P$. The concatenation argument again shows that $\operatorname{dim}_{k}(P \times Q) \leq \operatorname{dim}_{k}(P)+\operatorname{dim}_{k}(Q)$.

For $n \geq 3$, the poset $S_{n}$ is a subposet of the containment poset on the family of all subsets of $[n]$. Thus $S_{n}$ embeds in $\underline{2}^{n}$, and the dimension hierarchy collapses to yield $\operatorname{dim}_{k} S_{n}=n$ for $n \geq 3$ and $k \geq 2$. By a more detailed look at Trotter's counting argument for the lower bound, we determine $\operatorname{dim}_{k}\left(S_{m} \times S_{n}\right)$ whenever $\min \{m, n\} \geq 3$ and $k \geq 2$. Our main result in this direction is that $\operatorname{dim}_{k}\left(S_{m} \times S_{n}\right)=m+n-\min \{2, k-2\}$.

Our result suggests an extension of Trotter's conjecture. A poset is connected if its diagram is connected.

Conjecture 1.1 For connected posets $P$ and $Q$ with dimension at least $2, \operatorname{dim}_{k}(P \times Q) \geq$ $\operatorname{dim}_{k} P+\operatorname{dim}_{k} Q-2$.

We show in Section 2 that for disconnected posets no such conjecture holds, and the difference between $\operatorname{dim}_{k} P+\operatorname{dim}_{k} P$ and $\operatorname{dim}_{k}(P \times P)$ can be arbitrarily large.

The definition of dimension via embedding in products of chains is due to Hiraguchi [10] and to Ore [14]. It is equivalent to the earlier and more familiar definition of Dushnik and Miller [3] that in essence breaks down the embedding by coordinates. We will work with $k$-dimension in this phrasing.

A $k$-extension of $P$ is an order-preserving map from $P$ into $\underline{k}$. We view a $k$-extension $L$ as an ordered list of $k$ sets partitioning $P$; if $x<y$, then $y$ cannot be in an earlier set than $x$. A set of $k$-extensions is distinguishing if no two elements are given the same value in every $k$-extension in the set. A $k$-realizer of $P$ is a set $\left\{L_{1}, \ldots, L_{t}\right\}$ of $k$-extensions that is distinguishing and has the property that $x \leq y$ in $P$ if and only if $L_{i}(x) \leq L_{i}(y)$ for 
$1 \leq i \leq t$. The $k$-dimension of $P$ is the minimum size of a $k$-realizer of $P$. The coordinates in an embedding of $P$ in $\underline{k}^{t}$ yield a $k$-realizer of size $t$ for $P$, and vice versa.

Following Trotter [17], we count what can be accomplished by a single $k$-extension. A critical pair is an ordered pair $(x, y)$ of incomparable elements in $P$ such that $(z<x \Rightarrow z<y)$ and $(z>y \Rightarrow z>x)$. In other words, no relation can be added to $P$ that implies $x>y$. A critical pair $(x, y)$ is reversed in a $k$-extension $L$ if $x>y$ in $L$. A distinguishing set of $k$-extensions of $P$ is a realizer of $P$ if and only if every critical pair is reversed in at least one of its $k$-extensions.

Let $S(L)$ denote the set of critical pairs reversed in a $k$-extension $L$ of $P$. A $k$-extension $L$ of $P$ is saturated if $S(L)$ is maximal among all the sets $S\left(L^{\prime}\right)$ such that $L^{\prime}$ is a $k$-extension of $P$. A $k$-extension $L$ is dominated by a $k$-extension $L^{\prime}$ if $S(L) \subset S\left(L^{\prime}\right)$.

Lower bounds follow by counting the critical pairs of various types that can be reversed by saturated $k$-extensions. The requirement of reversing all the critical pairs yields a linear optimization problem giving a lower bound on the size of a $k$-realizer. Achieving the lower bound by constructing appropriate extensions completes the computation of the $k$-dimension. It is important to note that in proving lower bounds via such counting arguments, the condition that a $k$-realizer must distinguish the elements can be ignored.

In Section 6, we introduce another technique to give a simple proof of a stronger result about 2-dimension of iterated products of the standard examples: $\operatorname{dim}_{2}\left(\prod_{i=1}^{t} S_{n_{i}}\right)=\sum n_{i}$. Since products of the standard examples are not standard examples, this proves the conjecture about 2-dimension of products for a class of non-bounded posets that includes more than just the standard examples.

\section{Disconnected Posets}

Conjecture 1.1 is restricted to connected posets for the following reason:

Proposition 2.1 For the antichain $A_{n}$ with $n$ elements, the difference between $\operatorname{dim}_{2} A_{n}+$ $\operatorname{dim}_{2} A_{n}$ and $\operatorname{dim}_{2}\left(A_{n} \times A_{n}\right)$ grows without bound.

Proof. By Sperner's Theorem, the maximum size of an antichain of subsets of $[t]$ is $\left(\begin{array}{c}t \\ \lfloor t / 2\rfloor\end{array}\right)$. Hence $\operatorname{dim}_{2} A_{n}$ is the minimum $t$ such that $\left(\begin{array}{c}t \\ \lfloor t / 2\rfloor\end{array}\right) \geq n$. Thus $\operatorname{dim}_{2} A_{n} \in \lg n+\frac{1}{2} \lg \lg n+$ $O(\lg \lg \lg n)$, where $\lg$ denotes $\log _{2}$.

Note that $A_{n} \times A_{n}=A_{n^{2}}$. We have $\operatorname{dim}_{2} A_{n}+\operatorname{dim}_{2} A_{n} \in 2 \lg n+\lg \lg n+O(\lg \lg \lg n)$, but $\operatorname{dim}_{2}\left(A_{n} \times A_{n}\right) \in 2 \lg n+\frac{1}{2} \lg \lg n+O(\lg \lg \lg n)$. Hence the difference between $\operatorname{dim}_{2} A_{n}+$ $\operatorname{dim}_{2} A_{n}$ and $\operatorname{dim}_{2}\left(A_{n} \times A_{n}\right)$ can be arbitrarily large. 
We generalize Proposition 2.1 and its proof in two natural ways. First, we prove the claim also for $\operatorname{dim}_{k}$ (in fact, $k$ may grow slowly with $n$ ). Second, we show for $k=2$ that the computation remains valid when the antichain is replaced with multiple copies of any bounded poset. We cannot suppress $k$ and extend the construction to ordinary dimension, because $\operatorname{dim} A_{n^{2}}=2=\operatorname{dim} A_{n}+\operatorname{dim} A_{n}-2$.

For $k$-dimension, we mimic the argument for Proposition 2.1. As in that discussion, $\operatorname{dim}_{k}\left(A_{n}\right)$ is the smallest $t$ such that $\underline{k}^{t}$ contains $A_{n}$. Thus we need to approximate the width of $\underline{k}^{t}$. Since chain-products are symmetric chain orders, the width is the size of the middle rank; that is, the number of elements of $\{0, \ldots, k-1\}^{t}$ whose entries sum to $\lfloor t(k-1) / 2\rfloor$. The asymptotic behavior of the width is a special case of a result of Alekseev [1] that was generalized by Engel [4] (see [5]). We give a short argument for the special case needed here.

Lemma 2.2 For $k \in o\left(e^{t} / t^{1 / 2}\right)$, the width $w\left(\underline{k}^{t}\right)$ of $\underline{k}^{t}$ is asymptotic to $k^{t-1} / \sqrt{\pi t / 6}$.

Proof. It suffices to show that the probability of a uniformly selected random member of $\underline{k}^{t}$ having rank $\lfloor t(k-1) / 2\rfloor$ is asymptotic to $1 / \sqrt{\left(k^{2}-1\right) \pi t / 6}$. The rank $R_{t}$ is the sum of $t$ independent uniform random variables on $\{0, \ldots, k-1\}$; the mean $\mu$ and variance $\sigma^{2}$ of each are $(k-1) / 2$ and $\left(k^{2}-1\right) / 12$, respectively. We follow the proof of the Local Limit Theorem of Gnedenko [7] to show

$$
\lim _{t \rightarrow \infty}\left|\sqrt{t \sigma^{2}} \mathbb{P}\left(R_{t}=x\right)-\frac{1}{\sqrt{2 \pi}} e^{-\frac{(x-t \mu)^{2}}{2\left(t \sigma^{2}\right)}}\right|=0 .
$$

With $z=\frac{x-t \mu}{\sqrt{t \sigma^{2}}}$, it suffices to show $W_{t} \rightarrow 0$, where $W_{t}=2 \pi\left(\sqrt{t \sigma^{2}} \mathbb{P}\left(R_{t}=x\right)-\frac{1}{\sqrt{2 \pi}} e^{-z^{2} / 2}\right)$. Using change of variables and Fourier transformation, Gnedenko [7] proved

$$
W_{t}=\int_{-\pi \sqrt{t \sigma^{2}}}^{\pi \sqrt{t \sigma^{2}}} e^{-i x z-\frac{i x \mu}{\sqrt{t \sigma^{2}}}} f_{t}\left(\frac{x}{\sqrt{t \sigma^{2}}}\right) d x-\int_{-\infty}^{\infty} e^{-i x z-x^{2} / 2} d x
$$

where $f_{t}(s)=\sum_{l=-\infty}^{\infty} e^{-i s l} \mathbb{P}\left(R_{t}=s\right)$.

Given positive constants $A$ and $\epsilon$, for $t>\left(\frac{A}{\epsilon \sigma}\right)^{2}$ we write $W_{t}$ as the sum of four integrals:

$$
W_{t}=I_{1}+I_{2}+I_{3}+I_{4}
$$

where

$$
\begin{array}{ll}
I_{1}=\int_{-A}^{A} e^{-i x z}\left[e^{-\frac{i x \mu}{\sqrt{t \sigma^{2}}}} f_{t}\left(\frac{x}{\sqrt{t \sigma^{2}}}\right)-e^{-x^{2} / 2}\right] d x, & I_{2}=\int_{A \leq|x|<\epsilon \sqrt{t \sigma^{2}}} e^{-i x z-\frac{i x \mu}{\sqrt{t \sigma^{2}}}} f_{t}\left(\frac{x}{\sqrt{t \sigma^{2}}}\right) d x, \\
I_{3}=\int_{\epsilon \sqrt{t \sigma^{2}} \leq|x|<\pi \sqrt{t \sigma^{2}}} e^{-i x z-\frac{i x \mu}{\sqrt{t \sigma^{2}}}} f_{t}\left(\frac{x}{\sqrt{t \sigma^{2}}}\right) d x, & I_{4}=-\int_{|x|>A} e^{-i x z-x^{2} / 2} .
\end{array}
$$


Following Gnedenko and using $\left|e^{-i x z}\right|=1$, for fixed $A$ we have

$$
\left|I_{4}\right| \leq 2 \int_{A}^{\infty} e^{-x^{2} / 2} d x \leq \frac{2}{A} \int_{A}^{\infty} x e^{-x^{2} / 2} d x=\frac{2}{A} e^{\frac{-A^{2}}{2}}
$$

Since $R_{t}$ tends to a normally distributed variable as $t \rightarrow \infty$,

$$
e^{-\frac{i x \mu}{\sqrt{t \sigma^{2}}}} f_{t}\left(\frac{x}{\sqrt{t \sigma^{2}}}\right) \rightarrow e^{-x^{2} / 2}
$$

uniformly with respect to $x$, and thus $I_{1} \rightarrow 0$. For sufficiently small positive $\epsilon$, Taylor expansion yields the second inequality in the computation for $I_{2}$ :

$$
\left|I_{2}\right| \leq 2 \int_{A}^{\epsilon \sqrt{t \sigma^{2}}}\left|e^{-\frac{i x \mu}{\sqrt{t \sigma^{2}}}} f_{t}\left(\frac{x}{\sqrt{t \sigma^{2}}}\right)\right| d x \leq 2 \int_{A}^{\epsilon \sqrt{t \sigma^{2}}} e^{-x^{2} / 4} d x<2 \int_{A}^{\infty} e^{-x^{2} / 4} d x<\frac{4}{A} e^{-A^{2} / 4} .
$$

Therefore, by choosing $\epsilon$ small and $A$ large, we can make $I_{1}+I_{2}+I_{4}$ as small as desired. By another theorem of Gnedenko [8], there is a positive constant $c$ such that

$$
\left|e^{-\frac{i x \mu}{\sqrt{t \sigma^{2}}}} f_{t}\left(\frac{x}{\sqrt{t \sigma^{2}}}\right)\right| \leq e^{-t c}
$$

Thus

$$
\left|I_{3}\right| \leq \int_{\epsilon \sqrt{t \sigma^{2}} \leq|x| \leq \pi \sqrt{t \sigma^{2}}} e^{-t c} d x<2 \pi \sigma \sqrt{t} e^{-t c} .
$$

We obtain $\left|I_{3}\right| \rightarrow 0$ and $W_{t} \rightarrow 0$ as long as $\sigma \sqrt{t} e^{-t c} \rightarrow 0$. Since $\sigma=\sqrt{\left(k^{2}-1\right) / 12}$, the conclusion is valid when $k \in o\left(e^{t} / t^{1 / 2}\right)$.

We conclude that the probability of having rank $x$ is asymptotic to $e^{-(x-t \mu)^{2} /\left(2 t \sigma^{2}\right)} / \sqrt{2 \pi t \sigma^{2}}$. Setting $x=\lfloor t(k-1) / 2\rfloor$ completes the proof.

Using Lemma 2.2, we show that the $k$-dimension of antichains is arbitrarily subadditive. In fact, we obtain that conclusion even when $k$ grows (sufficiently slowly) with $n$.

Theorem 2.3 Viewing $k$ as a function of $n$, the difference between $2 \operatorname{dim}_{k}\left(A_{n}\right)$ and $\operatorname{dim}_{k}\left(A_{n} \times\right.$ $\left.A_{n}\right)$ grows without bound as $n$ grows, as long as $k \in o(\ln n)$.

Proof. Recall that $\operatorname{dim}_{k}\left(A_{n}\right)$ is the least $t$ such that $w\left(\underline{k}^{t}\right) \geq n$. By Lemma $2.2, \operatorname{dim}_{k}\left(A_{n}\right) \in$ $\log _{k} n+\frac{1}{2} \log _{k} \log _{k} n+O\left(\log _{k} \log _{k} \log _{k} n\right)$. Now $2 \operatorname{dim}_{k}\left(A_{n}\right)-\operatorname{dim}_{k}\left(A_{n} \times A_{n}\right)$ is given by the same computation as in Proposition 2.1, with $\log _{k}$ in place of $\lg$.

The resulting difference is $\frac{1}{2} \log _{k} \log _{k} n+O\left(\log _{k} \log _{k} \log _{k} n\right)$. Since $\log _{k} \log _{k} n=\frac{\ln \ln n-\ln \ln k}{\ln k}$, the difference is unbounded when $k=o(\ln n)$.

We now generalize Proposition 2.1 in a different direction by replacing the elements of the antichain $A_{n}$ with copies of any bounded poset $P$. 
Lemma 2.4 If $P$ is a bounded poset and $\operatorname{dim}_{2} P=p$, then $m P$ embeds in $\underline{2}^{t}$ if and only if $m\left(\underline{p+1)}\right.$ embeds in $\underline{2}^{t}$.

Proof. Consider an embedding of $m P$ into $\underline{2}^{t}$. Each copy of $P$ must embed into the interval between the images of its top and bottom elements. Each such interval is isomorphic to $\underline{2}^{s}$ for some $s$ with $p \leq s \leq t$. The top of copy $i$ of $P$ fails to be above the bottom of copy $j$ of $P$ if and only if no element of the $i$ th such interval is above any element of the $j$ th such interval. In particular, choosing a $(p+1)$-element chain from each of the intervals yields an embedding of $m\left(\underline{p+1)}\right.$ in $\underline{2}^{t}$.

Conversely, an embedding of $m(\underline{p+1})$ yields $m$ intervals of length at least $p$ in $\underline{2}^{t}$ that serve as the intervals into which copies of $P$ can be embedded to embed $m P$ into $\underline{2}^{t}$.

The problem of embedding $m(\underline{p+1})$ into $\underline{2}^{t}$ has a well-known solution.

Lemma 2.5 (Griggs-Stahl-Trotter [6]) $m(\underline{p+1})$ embeds in $\underline{2}^{t}$ if and only if $m \leq\left(\begin{array}{c}t-p \\ \lfloor(t-p) / 2\rfloor\end{array}\right)$.

Theorem 2.6 If $P$ is a bounded poset and $\operatorname{dim}_{2}(P)=p$, then

$$
\operatorname{dim}_{2} m P=\min \left\{t: \quad\left(\begin{array}{c}
t-p \\
\lfloor(t-p) / 2\rfloor
\end{array}\right) \geq m\right\} .
$$

In particular, $2 \operatorname{dim}_{2}(m P)-\operatorname{dim}_{2}(m P \times m P)$ is unbounded as a function of $m$.

Proof. The equation is the immediate consequence of the two preceding lemmas. We apply it also to $m P \times m P$. Note that $m P \times m P=m^{2}(P \times P)$; also, $\operatorname{dim}_{2}(P \times P)=2 \operatorname{dim}_{2} P=2 p$ since $P$ is a bounded poset. Therefore, $\operatorname{dim}_{2}(m P \times m P)=\min \left\{u:\left(\begin{array}{c}u-2 p \\ \lfloor(u-2 p) / 2\rfloor\end{array}\right) \geq m^{2}\right\}$.

As in Proposition 2.1, the least $x$ satisfying $\left(\begin{array}{c}x \\ \lfloor x / 2\rfloor\end{array}\right) \geq n$ is $\lg n+\frac{1}{2} \lg \lg n+O(\lg \lg \lg n)$. We thus compute

$$
\begin{aligned}
2 \operatorname{dim}_{2}(m P)-\operatorname{dim}_{2}(m P \times m P) \in & 2\left(p+\lg m+\frac{1}{2} \lg \lg m+O(\lg \lg \lg m)\right) \\
& -\left(2 p+\lg m^{2}+\frac{1}{2} \lg \lg m+O(\lg \lg \lg m)\right) \\
= & \frac{1}{2} \lg \lg m+O(\lg \lg \lg m) .
\end{aligned}
$$

We conclude that $2 \operatorname{dim}_{2}(m P)-\operatorname{dim}_{2}(m P \times m P)$ is unbounded as a function of $m$. 


\section{Critical Pairs in Products of Bipartite Posets}

A bipartite poset is a poset containing no 3-element chain, so that each element is maximal or minimal in the poset (equivalently, it is a poset whose comparability graph is bipartite). In this section we develop technical properties of bipartite posets that are needed for the reductions in our dimension arguments to be valid. The standard examples have these properties. We discuss the standard examples in the following form.

Definition 3.1 The standard example $S_{n}$ consists of minimal elements $\left\{a_{1}, \ldots, a_{n}\right\}$ and maximal elements $\left\{b_{1}, \ldots, b_{n}\right\}$, with $a_{i}<b_{j}$ if and only if $i \neq j$.

The critical pairs in $S_{n}$ are $\left\{\left(a_{i}, b_{i}\right): 1 \leq i \leq n\right\}$. Each linear extension reverses at most one such pair. On the other hand, $\operatorname{dim}_{2} S_{n} \leq n$ for $n \geq 3$, since $S_{n}$ is a subposet of $\underline{2}^{n}$. Thus $n \leq \operatorname{dim} S_{n} \leq \operatorname{dim}_{k} S_{n} \leq \operatorname{dim}_{2} S_{n} \leq n$, and equality holds throughout for all $k \geq 2$ and $n \geq 3$. This yields the trivial bound $\operatorname{dim}_{k}\left(S_{n} \times S_{m}\right) \leq n+m$.

In a product $P_{1} \times P_{2}$, we write any element $x$ as an ordered pair $\left(x_{1}, x_{2}\right)$. Note that $x$ is minimal if and only if $x_{1}$ is minimal in $P_{1}$ and $x_{2}$ is minimal in $P_{2}$ (similarly for maximal). We write $x \| y$ to indicate incomparability of $x$ and $y$.

Definition 3.2 A bipartite poset $P$ is robust if (1) whenever $x \| y$ in $P$ there is an element comparable to $x$ but not to $y$ (and similarly an element comparable to $y$ but not $x$ ), and (2) whenever $u<v$ in $P$ there are incomparable elements $z$ and $w$ with $z<v$ and $u<w$.

Lemma 3.3 If $P_{1}$ and $P_{2}$ are robust bipartite posets, then the critical pairs in $P_{1} \times P_{2}$ are the ordered incomparable pairs $(x, y)$ such that $x$ is minimal and $y$ is maximal in $P \times Q$.

Proof. By definition, the condition for $(x, y)$ to be a critical incomparable pair is that $(z<x \Rightarrow z<y)$ and $(z>y \Rightarrow z>x)$. If $x$ is minimal and $y$ is maximal, then these conditions hold vacuously. We show that $(x, y)$ is not a critical pair when $x$ is not minimal, and then by symmetry also $(x, y)$ is not a critical pair when $y$ is not maximal.

As discussed in Section 1, the pair $(x, y)$ is not critical if there is another incomparable pair $(z, w)$ such that adding $z>w$ to the order relation implies $x>y$ by transitivity. We seek an incomparable pair $(z, w)$ with $x>z$ and $w>y$.

If $x$ is not minimal, then $x_{1}$ or $x_{2}$ is not minimal in its factor. We may assume by symmetry that $x_{1}$ is not minimal in $P_{1}$. We will let $z=\left(z_{1}, x_{2}\right)$ and $w=\left(w_{1}, y_{2}\right)$, for suitable choices of $z_{1}$ and $w_{1}$.

If $y_{1}<x_{1}$, then robustness implies that $P_{1}$ has incomparable elements $z_{1}$ and $w_{1}$ such that $z_{1}<x_{1}$ and $y_{1}<w_{1}$. The only other case is $y_{1} \| x_{1}$ (since $x_{1}$ is not minimal). Now robustness guarantees $z_{1}$ such that $z_{1}<x_{1}$ and $z_{1} \| y_{1}$; with $w_{1}=y_{1}$, putting $\left(z_{1}, x_{2}\right)$ over $\left(w_{1}, y_{2}\right)$ again forces $x$ over $y$. 
Lemma 3.4 If $P_{1}$ and $P_{2}$ are robust bipartite posets with size at least 2 , then a family of $k$-extensions of $P_{1} \times P_{2}$ is a $k$-realizer if and only if it reverses all critical pairs.

Proof. Necessity is immediate. For sufficiency, a $k$-realizer must reverse all ordered incomparable pairs and must distinguish all pairs of elements. If $x$ and $y$ are incomparable in the product, then there is a critical pair $(u, v)$ such that $u \leq x$ and $y \leq v$. Putting $u$ above $v$ in some $k$-extension both reverses $(x, y)$ and distinguishes $x$ and $y$.

It remains to show that comparable pairs are distinguished. Suppose that $u<v$. Since chains in $P_{1} \times P_{2}$ have at most three elements, $u$ is minimal or $v$ is maximal; we assume by symmetry that $u$ is minimal. We consider two cases.

Case 1: $u$ is minimal and $v$ is maximal. By robustness in each factor, there are incomparable elements $z_{i}$ and $w_{i}$ with $z_{i}<v_{i}$ and $u_{i}<w_{i}$. Hence $z$ and $w$ are incomparable in the product, with $z$ minimal and $w$ maximal. By Lemma 3.3, $(z, w)$ is a critical pair. A $k$-extension that puts $z$ over $w$ also puts $v$ over $u$ and distinguishes $u$ and $v$.

Case 2: $u$ is minimal and $v$ is not maximal. By symmetry, we may assume that $v_{1}$ is minimal in $P_{1}$ and $v_{2}$ is maximal in $P_{2}$. Since $u<v$, we have $u_{1}=v_{1}$. Since $\left|P_{1}\right| \geq 2$, robustness yields $r_{1} \in P_{1}$ such that $r_{1}>u_{1}$. Since $u_{2}<v_{2}$, robustness of $P_{2}$ yields $z_{2}$ and $w_{2}$ such that $z_{2}<v_{2}$ and $u_{2}<w_{2}$. By Lemma 3.3, $\left(\left(u_{1}, z_{2}\right),\left(r_{1}, w_{2}\right)\right)$ is a critical pair. A $k$-extension that puts $\left(u_{1}, z_{2}\right)$ over $\left(r_{1}, w_{2}\right)$ also puts $v$ over $u$ and distinguishes $u$ and $v$.

Corollary 3.5 If $P_{1}$ and $P_{2}$ are robust bipartite posets with size at least 2 , then $\operatorname{dim}_{k}\left(P_{1} \times P_{2}\right)$ equals the $k$-dimension of the subposet $R$ of $P_{1} \times P_{2}$ consisting of the maximal elements and the minimal elements. Furthermore, $R$ is itself a robust bipartite poset.

Proof. Let $P=P_{1} \times P_{2}$. By Lemma 3.3, $R$ contains all elements of critical pairs in $P$. Also, $k$-extensions reversing them can be augmented to $k$-extensions of $P$ by iteratively giving an image to a missing element $x$ that is between the images of elements less than $x$ and the images of elements greater than $x$. By Lemma 3.4, this produces a $k$-realizer of $P$. Hence $\operatorname{dim}_{k}(P) \leq \operatorname{dim}_{k}(R)$, and equality holds by monotonicity.

For the second statement, $R$ is clearly bipartite. For robustness, consider $x, y \in R$ with $x=\left(x_{1}, x_{2}\right)$ and $y=\left(y_{1}, y_{2}\right)$. If $x<y$ in $R$, then $x_{1}<y_{1}$ and $x_{2}<y_{2}$. By robustness of the factors, for $i \in\{1,2\}$ there are incomparable $z_{i}, w_{i} \in P_{i}$ such that $z_{i}<y_{i}$ and $x_{i}<w_{i}$. Now $\left(z_{1}, z_{2}\right)$ and $\left(w_{1}, w_{2}\right)$ are incomparable, but $z<y$ and $x<w$.

Now suppose $x \| y$. Since coordinates of elements of $R$ are both maximal or both minimal in their factors, $x \| y$ cannot occur by $x_{1}<y_{1}$ and $x_{2}>y_{2}$; such elements are not in $R$. Hence by symmetry we may assume that incomparability occurs by $x_{1} \| y_{1}$. Now the robustness of $P_{1}$ guarantees $z \in P_{1}$ such that $z$ is comparable to $x_{1}$ but not $y_{1}$; the desired element is $\left(z, x_{2}\right)$.

Our results suggest the following extension of Conjecture 1.1: 
Conjecture 3.6 For robust bipartite posets $P$ and $Q$ with dimension at least $2, \operatorname{dim}_{k}(P \times$ $Q) \geq \operatorname{dim}_{k} P+\operatorname{dim}_{k} Q-\min \{2, k-2\}$. In particular, $\operatorname{dim}_{2}(P \times Q)=\operatorname{dim}_{2}(P)+\operatorname{dim}_{2}(Q)$.

Conjecture 3.6 does not extend to connected bipartite posets. The following example was provided by an anonymous reviewer.

Example 3.7 Let $R_{h}$ be the ranking consisting of a unique minimal element, $h$ maximal elements, and nothing else (the comparability graph of $R_{h}$ is a star with $h$ edges). We claim that $\operatorname{dim}_{2}\left(R_{3} \times R_{4}\right) \leq 6<7=\operatorname{dim}_{2}\left(R_{3}\right)+\operatorname{dim}_{2}\left(R_{4}\right)$. Note that $\operatorname{dim}_{2}\left(R_{3}\right)=3$ and $\operatorname{dim}_{2}\left(R_{4}\right)=4$ since $R_{h}$ has an antichain of size $h$.

For the upper bound on $\operatorname{dim}_{2}\left(R_{3} \times R_{4}\right)$, let the elements of $R_{3}$ be $p, p_{1}, p_{2}, p_{3}$ and the elements of $R_{4}$ be $q, q_{1}, q_{2}, q_{3}, q_{4}$, with $p$ and $q$ the minimal elements. To embed $R_{3} \times R_{4}$ in $\underline{2}^{6}$, first assign $(p, q)$ to $\varnothing$. Next, map $\left(p_{i}, q\right)$ to $A_{i}$, where $A_{1}=\{1,2\}, A_{2}=\{3,4\}$, and $A_{3}=\{5,6\}$. Also, map $\left(p, q_{i}\right)$ to $B_{i}$, where $B_{1}=\{1,3,5\}, B_{2}=\{1,4,6\}, B_{3}=\{2,3,6\}$, and $B_{4}=\{2,4,5\}$. These are the four sets with odd sum that contain one element from each of $A_{1}, A_{2}, A_{3}$. Finally, map $\left(p_{i}, q_{j}\right)$ to $A_{i} \cup B_{j}$.

It remains only to show that $A_{i} \cup B_{j}$ contains only $A_{i}$ and $B_{j}$ among these sets. Note that $\left|A_{i} \cup B_{j}\right|=4$, and the sets $A_{1}, A_{2}, A_{3}$ are pairwise disjoint. Since $A_{k} \nsubseteq B_{j}$, having $A_{k} \subseteq A_{i} \cup B_{j}$ requires $k=i$. Similarly, since $B_{j}$ and $B_{k}$ share exactly one element, their union has size 5 , so $B_{k} \subseteq A_{i} \cup B_{j}$ requires $k=j$.

An analogous construction shows that $\operatorname{dim}_{2}\left(R_{4} \times R_{8}\right) \leq \operatorname{dim}_{2}\left(R_{4}\right)+\operatorname{dim}_{2}\left(R_{8}\right)-1$, and in general $\operatorname{dim}_{2}\left(R_{h} \times R_{2^{h-1}}\right) \leq 2 k$. However, for $h \geq 5$ the value of $\operatorname{dim}_{2}\left(R_{h}\right)+\operatorname{dim}_{2}\left(R_{2^{h-1}}\right)$ is too small to extend Example 3.7 to an infinite family.

\section{Saturated $k$-extensions of $S_{m} \times S_{n}$}

For $n \geq 3, S_{n}$ is a robust bipartite poset, so Corollary 3.5 applies to $S_{m} \times S_{n}$. In $S_{m} \times S_{n}$, we write $a_{i, j}$ for the minimal element $\left(a_{i}, a_{j}^{\prime}\right)$, where $a_{i}$ and $a_{j}^{\prime}$ are the $i$ th and $j$ th minimal elements in $S_{m}$ and $S_{n}$, respectively. Similarly, $b_{r, s}$ denotes the maximal element $\left(b_{r}, b_{s}^{\prime}\right)$. A $\min / \max$ pair $\left(a_{i, j}, b_{r, s}\right)$ is incomparable (and hence a critical pair) if and only if $i=r$ or $j=s$. That is, $a_{i, j}<b_{r, s}$ in $S_{m} \times S_{n}$ if $i \neq r$ and $j \neq s$.

We view the minimal elements as forming one $m \times n$ grid and the maximal elements as forming another. Corresponding positions act as "pins" holding the two grids together. The incomparable pairs consist of one position from each grid, taken from the same column or from the same row. 
Definition 4.1 The critical pairs in $S_{m} \times S_{n}$, written as $\left(a_{i, j}, b_{r, s}\right)$, form three classes:

a) vertical pairs are those with $i \neq r$ and $j=s$,

b) horizontal pairs are those with $i=r$ and $j \neq s$,

c) straight pairs ("pins") are those with $i=r$ and $j=s$.

There are $m^{2} n-m n$ vertical pairs, $m n^{2}-m n$ horizontal pairs, and $m n$ straight pairs.

Let $S^{*}$ be the subposet consisting of the maximal and the minimal elements of $S_{m} \times S_{n}$. A 2-extension of $S^{*}$ is an order-preserving map from $S^{*}$ to $\{0,1\}$. We represent it as two 0,1 matrices, a lower one for the minimal elements and an upper one for the maximal elements. Let $\alpha_{i, j}$ denote the value assigned to $a_{i, j}$, and let $\beta_{r, s}$ denote the value assigned to $b_{r, s}$.

The lower bound for $\operatorname{dim}_{2}\left(S_{m} \times S_{n}\right)$ follows from Theorem 6.8 without characterizing saturated 2-extensions, but including this reduces the case analysis for 3-extensions.

Lemma 4.2 Every saturated 2-extension of $S^{*}$ reverses precisely all vertical and straight pairs in one column (Type $V$ ), or all horizontal and straight pairs in one row (Type H), or all critical pairs using one element (Type B), or the two horizontal and two vertical pairs formed from two minimal and two maximal elements in each of $S_{m}$ and $S_{n}$ (Type C).

Proof. Below are examples of the types of 2-extensions described in the statement, when $m=3$ and $n=4$. The two grids for a single 2-extension appear in the same column.

\begin{tabular}{|l|l|l|l|}
\hline 0 & 1 & 1 & 1 \\
\hline 0 & 1 & 1 & 1 \\
\hline 0 & 1 & 1 & 1 \\
\hline
\end{tabular}

\begin{tabular}{|l|l|l|l|}
\hline 1 & 1 & 1 & 1 \\
\hline 1 & 1 & 1 & 1 \\
\hline 0 & 0 & 0 & 0 \\
\hline
\end{tabular}

\begin{tabular}{|l|l|l|l|}
\hline 1 & 1 & 1 & 1 \\
\hline 1 & 1 & 1 & 1 \\
\hline 0 & 1 & 1 & 1 \\
\hline
\end{tabular}

\begin{tabular}{|l|l|l|l|}
\hline 0 & 1 & 1 & 1 \\
\hline 0 & 1 & 1 & 1 \\
\hline 0 & 0 & 0 & 0 \\
\hline
\end{tabular}

\begin{tabular}{|l|l|l|l|}
\hline 1 & 1 & 1 & 1 \\
\hline 1 & 0 & 1 & 1 \\
\hline 0 & 1 & 1 & 1 \\
\hline
\end{tabular}

\begin{tabular}{|l|l|l|l|}
\hline 1 & 0 & 0 & 0 \\
\hline 1 & 0 & 0 & 0 \\
\hline 1 & 0 & 0 & 0 \\
\hline
\end{tabular}

Type $V$

\begin{tabular}{|l|l|l|l|}
\hline 0 & 0 & 0 & 0 \\
\hline 0 & 0 & 0 & 0 \\
\hline 1 & 1 & 1 & 1 \\
\hline
\end{tabular}

Type $H$

\begin{tabular}{|l|l|l|l|}
\hline 1 & 0 & 0 & 0 \\
\hline 1 & 0 & 0 & 0 \\
\hline 1 & 1 & 1 & 1 \\
\hline
\end{tabular}

Type $B$

\begin{tabular}{|l|l|l|l|}
\hline 0 & 0 & 0 & 0 \\
\hline 0 & 0 & 0 & 0 \\
\hline 1 & 0 & 0 & 0 \\
\hline
\end{tabular}

Type $B$

\begin{tabular}{|l|l|l|l|}
\hline 0 & 0 & 0 & 0 \\
\hline 1 & 0 & 0 & 0 \\
\hline 0 & 1 & 0 & 0 \\
\hline
\end{tabular}

Type $C$

Since extensions are order-preserving,

$$
\alpha_{i, j} \leq \beta_{r, s} \text { when } i \neq r \text { and } j \neq s .
$$

A critical pair is reversed when $\alpha_{i, j}=1$ and $\beta_{r, s}=0$ with $i=r$ or $j=s$; it is horizontal (and in row $r$ ) when $i=r$, vertical (and in row $s$ ) when $j=s$, and straight when both equalities hold. By "greediness", we mean that we have reached a point where we can assign 1 to all remaining positions in the bottom grid and 0 to all remaining positions in the top grid, maximizing the number of reversed pairs. We index grid positions from the lower left.

Let $L$ be a saturated 2-extension of $S^{*}$. If no horizontal or vertical pair is reversed, then a reversed pair has the form $\left(a_{i, j}, b_{i, j}\right)$, with $\alpha_{i, j}=1$ and $\beta_{i, j}=0$. Since no horizontal or 
vertical pair is reversed, by $(*)$ no other pair is reversed. Now $L$ is dominated by extensions of all types listed and is not saturated.

Suppose that $L$ reverses a horizontal pair; by symmetry, we may let it be $\left(a_{1,2}, b_{1,1}\right)$. Thus $\alpha_{1,2}=1$ and $\beta_{1,1}=0$. By $(*)$, we have $\beta_{i, j}=1$ for $i \neq 1$ and $j \neq 2$, and $\alpha_{i, j}=0$ for $i \neq 1$ and $j \neq 1$. If there is another reversed horizontal pair in the first row of the form $\left(a_{1, s}, b_{1, t}\right)$ with $s \neq 2$ and $t \neq 1$, then the upper grid is all 1 outside row 1 and the lower grid is all 0 outside row 1. By greediness, saturation requires that $L$ is Type $H$, reversing all horizontal and straight pairs in a single row. Similarly, if disjoint vertical pairs in the same column are reversed, then $L$ is Type $V$.

If there is another reversed horizontal pair outside the first row, then the constraints imposed by the first pair require it to be $\left(a_{r, 1}, b_{r, 2}\right)$ with $r>1$. By symmetry, let $r=2$, so we have $\alpha_{2,1}=1$ and $\beta_{2,2}=0$.) Now $(*)$ forces $L$ to be Type $C$. The same conclusion holds when $L$ reverses vertical pairs in distinct columns.

In the remaining case, all reversed horizontal pairs lie in the same row, and all reversed vertical pairs lie in the same column. Suppose first that there is at least one of each type. By symmetry, we may assume that $\left(a_{1,2}, b_{1,1}\right)$ is a reversed horizontal pair. For each column $s$, either $\alpha_{r, s}=0$ for $r>1$ or $\beta_{r, s}=1$ for $r>1$, or both if $s>2$. Hence a reversed vertical pair must be in column 1 and use $b_{1,1}$ or be in column 2 and use $a_{1,2}$. In the first case, greediness now forces $L$ to be Type $B$, reversing all critical pairs using $b_{1,1}$. In the second case, again $L$ is Type $B$, reversing all critical pairs using $a_{1,2}$.

Finally, we have horizontal pairs in one row and no vertical pairs, or vertical pairs in one column and no horizontal pairs. By greediness, $L$ is Type $H$ or Type $V$, respectively.

In a $k$-extension, we partition the elements of $S^{*}$ into $k$ sets $X_{0}, \ldots, X_{k-1}$ We still represent such extensions using two grids, but with entries in $\{0, \ldots, k-1\}$. Still $(*)$ holds: $\alpha_{i, j} \leq \beta_{r, s}$ when $i \neq r$ and $j \neq s$.

Lemma 4.3 Every saturated 3-extension of $S^{*}$ reverses precisely all vertical and straight pairs in one column and the horizontal pairs using one element in that column (Type V), or all horizontal and straight pairs in one row and the vertical pairs using one element in that row (Type H), or all critical pairs using the elements in one critical pair (Type B), or all critical pairs using one element plus one more horizontal and one more vertical pair (Type C).

Proof. Below we show examples of the types of 3-extensions described in the statement when $m=3$ and $n=4$. Within Type $B$ we identify two subtypes $B_{1}$ and $B_{2}$. Compared to Type $B_{1}$, Type $B_{2}$ reverses one less horizontal pair and one more straight pair.

Let $L$ be a saturated 3-extension of $S^{*}$. If $L$ puts no 2 in the bottom grid, then changing every 2 into a 1 in the top grid yields a 2-extension with the same critical pairs. However, 


\begin{tabular}{|l|l|l|l|}
\hline 0 & 2 & 2 & 2 \\
\hline 0 & 2 & 2 & 2 \\
\hline 0 & 1 & 1 & 1 \\
\hline 1 & 0 & 0 & 0 \\
\hline 1 & 0 & 0 & 0 \\
\hline 2 & 0 & 0 & 0 \\
\hline
\end{tabular}

Type $V$

\begin{tabular}{|l|l|l|l|}
\hline 1 & 2 & 2 & 2 \\
\hline 1 & 2 & 2 & 2 \\
\hline 0 & 2 & 2 & 2 \\
\hline 2 & 0 & 0 & 0 \\
\hline 2 & 0 & 0 & 0 \\
\hline 2 & 1 & 1 & 1 \\
\hline
\end{tabular}

Type $V$

\begin{tabular}{|l|l|l|l|}
\hline 1 & 2 & 2 & 2 \\
\hline 1 & 2 & 2 & 2 \\
\hline 0 & 0 & 0 & 0 \\
\hline 0 & 0 & 0 & 0 \\
\hline 0 & 0 & 0 & 0 \\
\hline 2 & 1 & 1 & 1 \\
\hline
\end{tabular}

Type $H$

\begin{tabular}{|l|l|l|l|}
\hline 2 & 2 & 2 & 2 \\
\hline 2 & 2 & 2 & 2 \\
\hline 0 & 1 & 1 & 1 \\
\hline
\end{tabular}

\begin{tabular}{|l|l|l|l|}
\hline 1 & 0 & 0 & 0 \\
\hline 1 & 0 & 0 & 0 \\
\hline 2 & 2 & 2 & 2 \\
\hline
\end{tabular}

Type $H$

\begin{tabular}{|l|l|l|l|}
\hline 1 & 2 & 2 & 2 \\
\hline 1 & 2 & 2 & 2 \\
\hline 0 & 1 & 1 & 1 \\
\hline 1 & 0 & 0 & 0 \\
\hline 1 & 0 & 0 & 0 \\
\hline 2 & 1 & 1 & 1 \\
\hline
\end{tabular}

Type $B_{1}$

\begin{tabular}{|l|l|l|l|}
\hline 1 & 2 & 2 & 2 \\
\hline 1 & 2 & 2 & 2 \\
\hline 1 & 0 & 1 & 1 \\
\hline 0 & 1 & 0 & 0 \\
\hline 0 & 1 & 0 & 0 \\
\hline 2 & 1 & 1 & 1 \\
\hline
\end{tabular}

Type $B_{2}$

\begin{tabular}{|l|l|l|l|}
\hline 2 & 2 & 2 & 2 \\
\hline 1 & 2 & 2 & 2 \\
\hline 2 & 0 & 2 & 2 \\
\hline
\end{tabular}

\begin{tabular}{|l|l|l|l|}
\hline 0 & 1 & 0 & 0 \\
\hline 0 & 2 & 0 & 0 \\
\hline 2 & 1 & 1 & 1 \\
\hline
\end{tabular}

Type $C$

each 2-extension in Lemma 4.2 is strictly dominated by some 3-extension shown above. Therefore, $L$ puts a 2 in the bottom grid. Similarly, $L$ puts a 0 in the top grid. By (*), a 2 in the bottom grid and 0 in the top grid must be in the same row or in the same column and correspond to a reversed critical pair. We call such a reversed pair an extreme pair.

Suppose first that there is an extreme horizontal pair; we may assume by symmetry that $\alpha_{1,1}=2$ and $\beta_{1,2}=0$. By $(*)$, we have $\beta_{r, s}=2$ for $r \neq 1$ and $s \neq 1$, and we have $\alpha_{i, j}=0$ for $i \neq 1$ and $j \neq 2$. This implies that the only vertical pairs that can be reversed are those using $a_{1,1}$ or $b_{1,2}$. Also, the only horizontal pairs that can be reversed are those in the first row and $\left(a_{i, 2}, b_{i, 1}\right)$ with $i>1$.

If $2 \geq \alpha_{i, 2}>\beta_{i, 1} \geq 0$ with $i>1$, then $\alpha_{i, 2}=2$ or $\beta_{i, 1}=0$. The former forces the first column (except $\beta_{i, 1}$ ) and first row (except $\beta_{1,2}$ ) in the top grid to be 2 , while the latter forces the second column (except $\alpha_{i, 2}$ ) and first row (except $\alpha_{1,1}$ ) in the bottom grid to be 0 . If $\alpha_{i, 2}=2$, then the only possible reversed pairs now are those using $b_{1,2}$ plus two pairs using $b_{i, 1}$. If $\beta_{i, 1}=0$, then the only reversed pairs are those using $a_{1,1}$ plus two pairs using $a_{i, 2}$. By greediness, the extension now has Type $C$. Compared to Type $B_{2}$, we gain one horizontal pair, but we lose $n-2$ horizontal pairs, $m-2$ vertical pairs, and one straight pair. Type $C$ reverses only $m$ vertical pairs, $n$ horizontal pairs, and one straight pair.

Hence we may assume that no horizontal pair outside row 1 is reversed, so $\beta_{i, 1} \geq \alpha_{i, 2}$ for $i \neq 1$. If vertical pairs using both $a_{1,1}$ and $b_{1,2}$ are reversed, by $\alpha_{1,1}>\beta_{i, 1}$ and $\alpha_{r, 2}>\beta_{1,2}$ (possibly $i=r$ ), then $2 \geq \alpha_{1,1}>\beta_{i, 1} \geq \alpha_{r, 2}>\beta_{1,2} \geq 0$. Hence $\beta_{i, 1}=\alpha_{r, 2}=1$. Now $\beta_{i, 1}=1$ forces the first row (except $\alpha_{1,1}$ ) and second column (except $\alpha_{i, 2}$ ) of the bottom grid to be at most 1 , and $\alpha_{r, 2}=1$ forces the first row (except $\beta_{1,2}$ ) and first column of the top grid (except $\beta_{r, 1}$ ) to be at least 1 . By greediness, we set all these values to 1 , reversing precisely all critical pairs using $a_{1,1}$ and $b_{1,2}$. Now $L$ is Type $B_{2}$ (with two reversed straight pairs).

If reversed vertical pairs use at most one of $\left\{a_{1,1}, b_{1,2}\right\}$, then we may assume that no reversed vertical pair uses $b_{1,2}$. Since $\beta_{1,2}=0$, this implies that $\alpha_{i, 2}=0$ for $i \neq 1$. Now the only pairs that can be reversed are those in the first row and those in the first column that use $a_{1,1}$. We can reverse all of them by setting $\alpha_{1, j}=1$ for $j \neq 1$ and $\beta_{r, 1}=1$ for $r \neq 1$. The result is a 3-extension of Type $H$. 
This eliminates extreme horizontal pairs. For an extreme vertical pair, the argument is symmetric. Hence we may assume that there is one extreme straight pair and no other extreme pair (two extreme straight pairs would force an extreme horizontal or vertical pair).

By symmetry, we may assume that $\alpha_{1,1}=2$ and $\beta_{1,1}=0$. By $(*)$, the top grid is 2 outside the first row and column, and the bottom grid is 0 outside the first row and column. Since there are no extreme horizontal or vertical pairs, the remaining entries are at most 1 in the bottom grid and at least 1 in the top grid. Greediness sets them all equal to 1 and yields an extension of Type $B_{1}$ (with one reversed straight pair).

We treat all $k$ with $k \geq 4$ simultaneously. This means that we analyze what sets of critical pairs can be reversed for linear extensions, and we show that the maximal such sets can be achieved using 4-extensions. This yields $\operatorname{dim}_{k}\left(S_{m} \times S_{n}\right)=\operatorname{dim}\left(S_{m} \times S_{n}\right)$ for all $k \geq 4$. The analysis of linear extensions follows the approach of Trotter [17]; the main difference is that we separate horizontal and vertical pairs.

Lemma 4.4 Every saturated linear extension of $S^{*}$ (or saturated $k$-extension for $k \geq 4$ ) reverses precisely all vertical and straight pairs in one column and all horizontal pairs using two fixed elements (one maximal and one minimal) in that column (Type $V$ ), or all horizontal and straight pairs in one row and all vertical pairs using two fixed elements (one maximal and one minimal) in that row (Type H), or all critical pairs using the elements of one vertical pair or of one horizontal pair (plus one more critical pair) (Type B).

Proof. Below are examples of 4-extensions of these types, when $m=3$ and $n=4$. For Type $H$, the minimal and maximal elements that cover the vertical pairs may form a straight pair or a horizontal pair; both variations are shown. Analogous variations exist for Type $V$. In the examples for Type $B$, all critical pairs using $a_{1,1}$ are reversed, along with those using $b_{2,1}$ in the first example and those using $b_{1,2}$ in the second example, and the extra reversed pair puts $a_{2,2}$ over $b_{1,2}$ or $b_{2,1}$, respectively.

\begin{tabular}{|l|l|l|l|}
\hline 1 & 3 & 3 & 3 \\
\hline 1 & 3 & 3 & 3 \\
\hline 0 & 2 & 2 & 2 \\
\hline 2 & 0 & 0 & 0 \\
\hline 2 & 0 & 0 & 0 \\
\hline 3 & 1 & 1 & 1 \\
\hline
\end{tabular}

Type $V$

\begin{tabular}{|l|l|l|l|}
\hline 2 & 3 & 3 & 3 \\
\hline 2 & 3 & 3 & 3 \\
\hline 0 & 1 & 1 & 1 \\
\hline
\end{tabular}

\begin{tabular}{|l|l|l|l|}
\hline 1 & 0 & 0 & 0 \\
\hline 1 & 0 & 0 & 0 \\
\hline 3 & 2 & 2 & 2 \\
\hline
\end{tabular}

Type $H$

\begin{tabular}{|l|l|l|l|}
\hline 2 & 3 & 3 & 3 \\
\hline 2 & 3 & 3 & 3 \\
\hline 1 & 0 & 1 & 1 \\
\hline
\end{tabular}

\begin{tabular}{|l|l|l|l|}
\hline 0 & 1 & 0 & 0 \\
\hline 0 & 1 & 0 & 0 \\
\hline 3 & 2 & 2 & 2 \\
\hline
\end{tabular}

Type $H$

\begin{tabular}{|l|l|l|l|}
\hline 2 & 3 & 3 & 3 \\
\hline 0 & 3 & 3 & 3 \\
\hline 2 & 1 & 2 & 2 \\
\hline
\end{tabular}

\begin{tabular}{|l|l|l|l|}
\hline 1 & 0 & 0 & 0 \\
\hline 1 & 2 & 1 & 1 \\
\hline 3 & 0 & 0 & 0 \\
\hline
\end{tabular}

Type $B$

\begin{tabular}{|l|l|l|l|}
\hline 2 & 3 & 3 & 3 \\
\hline 1 & 3 & 3 & 3 \\
\hline 2 & 0 & 2 & 2 \\
\hline
\end{tabular}

\begin{tabular}{|l|l|l|l|}
\hline 0 & 1 & 0 & 0 \\
\hline 0 & 2 & 0 & 0 \\
\hline 3 & 1 & 1 & 1 \\
\hline
\end{tabular}

Type $B$ 
The above examples generalize for all $m$ and $n$ to show that extensions for $k \geq 4$ do exist that reverse the pairs as described in the statement.

Let $L$ be a saturated linear extension of $S^{*}$. A saturated extension must reverse some critical pair. By symmetry, we may assume that $a_{1,1}$ is the highest element on the extension that belongs to any reversed critical pair. Let $b_{r, s}$ be the lowest element in any reversed critical pair. Since $\alpha_{1,1}>\beta_{r, s}$, by $(*)$ we conclude that $r=1$ or $s=1$.

Case 1: $r=s$. That is, $r=s=1$. By $(*)$, we have $\beta_{p, q}>\alpha_{1,1}>\beta_{1,1}>\alpha_{i, j}$ when $1 \notin\{p, q, i, j\}$. Hence all reversed critical pairs use only elements in the first row or only in the first column of the grids. If some such pair uses neither $a_{1,1}$ nor $b_{1,1}$, then by symmetry we may assume that it is $\left(a_{1, j}, b_{1, q}\right)$, with $\alpha_{1, j}>\beta_{1, q}$. Since $j \neq 1$ and $q \neq 1$, by $(*)$ we have $\beta_{p, 1}>\alpha_{1, j}>\beta_{1, q}>\alpha_{i, 1}$ whenever $p \neq 1$ and $i \neq 1$. Hence there are no reversed pairs in the first column that avoid the lower-left elements. By greediness, we can now reverse all remaining pairs, which are the horizontal and straight pairs in the first row and all the critical pairs using $a_{1,1}$ and $b_{1,1}$. The result is an extension of Type $H$ (Type $V$ arises symmetrically).

Case 2: $r \neq s$. By symmetry, we may assume that the lowest element on the extension that belongs to any reversed pair is $b_{1,2}$. Again $(*)$ restricts the possible reversed pairs, since $\alpha_{1,1}>\beta_{1,2}$. All elements of the top grid not in the first row or column are above all elements of the bottom grid not in the first row or second column, so additional reversed pairs are confined to the first row or to the first two columns.

In particular, putting $a_{i, j}$ over $b_{r, s}$ requires $i=1$ or $j=2$, (due to $b_{1,2}$ ) and $r=1$ or $s=1$ (due to $a_{1,1}$ ), and $i=r$ or $j=s$. With $i=r$, the possible reversals are $\alpha_{1, j}>\beta_{1, s}$ and $\alpha_{i, 2}>\beta_{i, 1}$. With $j=s$, the possibilities are $\alpha_{1,1}>\beta_{r, 1}$ and $\alpha_{i, 2}>\beta_{1,2}$.

If $\alpha_{i, 2}>\beta_{i, 1}$ with $i \neq 1$, then $\beta_{p, 1}>\alpha_{i, 2}>\beta_{i, 1}>\alpha_{p, 2}$ for $p \neq i$ (although $\beta_{p, 1}<\alpha_{1,1}$ and $\alpha_{p, 2}>\beta_{1,2}$ remain possible), so this can occur for only one value of $i$. It if occurs, then $\beta_{1, s}>\alpha_{i, 2}>\beta_{i, 1}>\alpha_{1, j}$ for $s \neq 2$ and $j \neq 1$. Hence we obtain only one additional reversed pair $\left(a_{i, j}, b_{r, s}\right)$ with $i=r$. By greediness, we may have all of the remaining possibilities $\alpha_{1,1}>\beta_{r, 1}$ and $\alpha_{i, 2}>\beta_{1,2}$. The result is an extension of Type $B$. (The argument is symmetric when the lowest element of reversed pairs is $b_{2,1}$, again yielding Type $B$.)

Finally, if $\alpha_{i, 2}<\beta_{i, 1}$ whenever $i \neq 1$, then by greediness we may have $\alpha_{1, j}>\beta_{1, s}$ for all $j$ and $s$, and $\alpha_{1,1}>\beta_{r, 1}$ for all $r$, and $\alpha_{i, 2}>\beta_{1,2}$ for all $i$. The result is an extension of Type $H$ (it yields Type $V$ if the lowest element in a reversed pair is in column 1 ). 


\section{$5 \quad k$-dimension of $S_{m} \times S_{n}$}

For upper bounds, we use of Lemma 3.4 and Corollary 3.5. They guarantee that the constructions reversing the critical pairs in $S_{m} \times S_{n}$ yield $k$-realizers of the same size.

Theorem 5.1 If $m, n \geq 3$, then $\operatorname{dim}_{k}\left(S_{m} \times S_{n}\right)=m+n-\min \{2, k-2\}$.

Proof. For the upper bounds, we show that all critical pairs can be reversed using the desired number of $k$-extensions of types described in Lemmas 4.2, 4.3, and 4.4. For $k=2$, we use an extension of Type $V$ in each column and an extension of Type $H$ in each row to reverse all critical pairs using $m+n$ extensions.

For $k=3$, we use an extension of Type $H$ in each row and an extension of Type $V$ in columns 2 through $n$. In the Type $H$ extension for row $i$, we use $\alpha_{i, 1}=2$ and $\beta_{i, 1}=0$ and put $\beta_{r, 1}=1$ for all $r$ not equal to $i$, thereby reversing all the vertical and straight pairs using $a_{i, 1}$. Since every critical pair contained in the first column uses $a_{i, 1}$ for some $i$, these extensions of Type $H$ together reverse all critical pairs contained in the first column. Hence the remaining critical pairs are reversed by Type $V$ extensions in columns 2 through $n$.

For $k=4$, we use an extension of Type $H$ in each row and an extension of Type $V$ in columns 3 through $n$. In the Type $H$ extension for row $i$, we use $\alpha_{i, 1}=3$ and $\beta_{i, 2}=0$, thereby reversing all the vertical and straight pairs using $a_{i, 1}$ and $b_{i, 2}$. Since every critical pair contained in the first column uses $a_{i, 1}$ for some $i$, and every critical pair contained in the second column uses $b_{i, 2}$ for some $i$, these extensions of Type $H$ together reverse all vertical and straight pairs contained in the first two columns. Hence the remaining critical pairs are reversed by Type $V$ extensions in columns 3 through $n$.

For $k>4$, the upper bound follows from the construction for $k=4$, using extensions of the subposets induced by the sets $X_{0}, X_{1}, X_{2}, X_{3}$ on 4-extensions.

Now consider the lower bounds. For $k \in\{2,3,4\}$, Lemmas 4.2, 4.3, and 4.4 show that saturated $k$-extensions are of the types we have called $V, H, B, C$ (or when $k=3$ reverse strictly fewer critical pairs than one of these types). The numbers of critical pairs of each class that are reversed by each type of saturated $k$-extension appear in Table 1.

Table 1

\begin{tabular}{c|ccc|ccc|ccc} 
& & & & & & & \\
& & $k=2$ & & & & \\
& & & & & & \\
vertical & horiz. & straight & vertical & horiz. & straight & vertical & horiz. & straight \\
$V$ & $m^{2}-m$ & 0 & $m$ & $m^{2}-m$ & $n-1$ & $m$ & $m^{2}-m$ & $2 n-2$ & $m$ \\
$H$ & 0 & $n^{2}-n$ & $n$ & $m-1$ & $n^{2}-n$ & $n$ & $2 m-2$ & $n^{2}-n$ & $n$ \\
$B$ & $m-1$ & $n-1$ & 1 & $2 m-2$ & $2 n-2$ & 1 & $2 m-2$ & $2 n-2$ & 2 \\
$C$ & 2 & 2 & 0 & $m$ & $n$ & 1 & & &
\end{tabular}


When $k=3$, Type $B$ has variations $B_{1}$ and $B_{2}$, where $B_{1}$ appears in the table and $B_{2}$ reverses one more straight pair and one less horizontal or vertical pair than $B_{1}$.

These values yield the desired lower bounds numerically. We minimize the total number of saturated extensions subject to obtaining enough reversed critical pairs in each class. The result is a linear program. To obtain a lower bound, it suffices to exhibit a feasible solution to the dual problem that has at least the desired value. Ad hoc argument with variables for the number of extensions of each type also works.

The dual, with variables $v, h, s$, is to maximize the objective $n m(m-1) v+m n(n-1) h+$ mns subject to a linear constraint from each row of Table 1. For example, the constraint when $k=2$ for row $V$ is $m(m-1) v+0 h+m s \leq 1$ (the constant 1 comes from the objective of minimizing the sum of the number of extensions).

When $k=2$, we set $(v, h, s)=\left(\frac{1}{m(m-1)}, \frac{1}{n(n-1)}, 0\right)$ to obtain a feasible solution with objective value $m+n$. Hence $m+n$ such extensions are needed. Since $m, n \geq 3$, the constraints for Types $B$ and $C$ are not tight, corresponding to using only Types $V$ and $H$ in the upper bound construction.

When $k=3$, we set $(v, h, s)=\left(\frac{n-1}{(m n-1)(m-1)}, \frac{m-1}{(m n-1)(n-1)}, 0\right)$ to obtain a feasible solution with objective value $(m+n-2) \frac{m n}{m n-1}$. Since $(m+n-2) \frac{m n}{m n-1}>m+n-2$ and the number of 3 -extensions is an integer, we obtain $m+n-1$ as a lower bound. Again the constraints for Types $B_{1}, B_{2}$, and $C$ are not tight and only Types $V$ and $H$ are used in the construction.

When $k \geq 4$, we obtain the bound more directly. If $V+H+B<m+n-2$, then $V \leq n-2$ or $H \leq m-2$. By symmetry, we may assume that $V=n-r$ with $r \geq 2$. Now reversing the remaining $m(m-1) r$ vertical pairs requires $H+B \geq r m / 2>m+r-3$. Thus $V+H+B \geq n+m-2$.

\section{2-dimension of $t$-fold Products}

A different approach to 2-dimension avoids describing saturated extensions.

Definition 6.1 Critical pairs $(x, y)$ and $\left(x^{\prime}, y^{\prime}\right)$ are $k$-conflicted if every $k$-extension reverses at most one of them. A set $S$ of critical pairs is $k$-conflicted if every two critical pairs in $S$ are $k$-conflicted.

Remark 6.2 If $S$ is a $k$-conflicted set of critical pairs in a poset $P$, then $\operatorname{dim}_{k}(P) \geq|S|$.

Lemma 6.3 In a poset $P$, critical pairs $(x, y)$ and $\left(x^{\prime}, y^{\prime}\right)$ are 2-conflicted if $x<y^{\prime}$ or $x^{\prime}<y$. If $k \geq 3$, then $(x, y)$ and $\left(x^{\prime}, y^{\prime}\right)$ are $k$-conflicted if $x<y^{\prime}$ and $x^{\prime}<y$. 
Proof. If $x<y^{\prime}$, then reversing $(x, y)$ and $\left(x^{\prime}, y^{\prime}\right)$ in a $k$-extension $f$ requires $f(y)<f(x) \leq$ $f\left(y^{\prime}\right)<f\left(x^{\prime}\right)$. This uses three distinct values in $f$, so it cannot occur when $k=2$. If also $x^{\prime}<y$, then $f\left(x^{\prime}\right) \leq f(y)$ is needed, and this cannot be achieved for any $k$.

In dimension theory for posets, the condition $x<y^{\prime}$ and $x^{\prime}<y$ when $k \geq 3$ makes the pair $\left\{(x, y),\left(x^{\prime}, y^{\prime}\right)\right\}$ what is known as an "alternating cycle" of size 2 . The fact that a weaker condition suffices for pairs to be 2-conflicted is the reason why this notion is effective in proving lower bounds for 2-dimension. In order to motivate the argument for higher products, we first give a short explanation of it for $S_{m} \times S_{n}$.

Example 6.4 We show that if $m, n \geq 3$, then $\operatorname{dim}_{2}\left(S_{m} \times S_{n}\right)=m+n$. It suffices to present a 2-conflicted set of size $m+n$. Note that $\left(a_{i, j}, b_{r, s}\right)$ with $i=r$ or $j=s$ and $\left(a_{g, h}, b_{p, q}\right)$ with $g=p$ or $h=q$ are 2-conflicted if $a_{i, j}<b_{p, q}$ or $a_{g, h}<b_{r, s}$; that is, if $(i \neq p$ and $j \neq q)$ or $(g \neq r$ and $h \neq s)$.

We represent the order relations in one grid using arrows: $(i, j) \rightarrow(r, s)$ represents the pair $\left(a_{i, j}, b_{r, s}\right)$. The pair is incomparable if the arrow lies within one row or within one column. Two incomparable pairs are 2-conflicted if, as arrows, the tail of one is in a different row and different column from the head of the other. Figure 1 illustrates a 2-conflicted set of arrows. The size of the set is $6+(m-3)+(n-3)$, as desired.

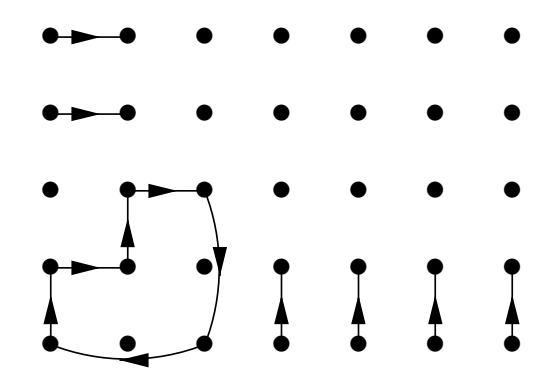

Figure 1: A 2-conflicted set for $S_{5} \times S_{7}$

Let $P=\prod_{i=1}^{t} S_{n_{i}}$. Let $A$ be the set of $t$-tuples whose $i$ th coordinate is minimal in $S_{n_{i}}$ for all $i$, and let $B$ be the set of $t$-tuples whose $i$ th coordinate is maximal in $S_{n_{i}}$ for all $i$. By Corollary 3.5, it follows by induction on $t$ that $\operatorname{dim}_{k} P=\operatorname{dim}_{k} R$, where $R$ is the subposet of $P$ with elements $A \cup B$. Represent each pair $\left(a_{i_{1}, \ldots, i_{t}}, b_{r_{1}, \ldots, r_{t}}\right)$ in $A \times B$ by an arrow from $\left(i_{1}, \ldots, i_{t}\right)$ to $\left(r_{1}, \ldots, r_{t}\right)$ in a $t$-dimensional grid. The pair is a critical pair if the head and tail agree in some coordinate. Two pairs are 2-conflicted if the tail of one differs from the head of the other in every coordinate. 
As motivated by Example 6.4, the idea of the proof for $t \geq 3$ is to first study $\prod_{i=1}^{t} S_{t}$, presenting a cycle $C_{t}$ of length $t^{2}$ in the $t$-dimensional grid, with the edge set forming a 2 -conflicted set. This yields $\operatorname{dim}_{2}\left(\prod_{i=1}^{t} S_{t}\right)=t \cdot t$. For $\prod_{i=1}^{t} S_{n_{i}}$ with each $n_{i} \geq t$, we then add $n_{i}-t$ arrows for extra hyperplanes in the $i$ th direction, copying arrows on $C_{t}$. This will yield $\operatorname{dim}_{2}\left(\prod_{i=1}^{t} S_{n_{i}}\right)=\sum_{i=1}^{t} n_{i}$.

Definition 6.5 The t-lagging cycle is the cycle $C_{t}$ consisting of vectors $v_{1}, \ldots, v_{t^{2}}$ in $[t]^{t}$ formed by letting $v_{1}=(1, \ldots, 1)$ and, for $j \geq 1$, forming $v_{j+1}$ from $v_{j}$ by adding 1 (modulo $t$ ) to each coordinate except the $i$ th, where $i \equiv j \bmod t$.

For example, $C_{4}$ is (reading down columns):

$$
\begin{array}{llll}
(1,1,1,1) & (4,4,4,4) & (3,3,3,3) & (2,2,2,2) \\
(1,2,2,2) & (4,1,1,1) & (3,4,4,4) & (2,3,3,3) \\
(2,2,3,3) & (1,1,2,2) & (4,4,1,1) & (3,3,4,4) \\
(3,3,3,4) & (2,2,2,3) & (1,1,1,2) & (4,4,4,1)
\end{array}
$$

Proposition 6.6 The t-lagging cycle $C_{t}$ has the following properties:

(1) In $t^{2}$ steps of the $t$-lagging cycle, each entry augments $t^{2}-t$ times, and $C_{t}$ is a cycle.

(2) Each vector in the cycle has the form $(q-1, \ldots, q-1, q, \ldots, q)$ for some $q$ (modulo $t$ ), every such vector arises, and each $v_{j}$ agrees with $v_{j+1}$ in the position of the first $q$ in $v_{j}$.

(3) Each arrow $v_{j} \rightarrow v_{j+1}$ for successive elements of the cycle corresponds to an incomparable $\operatorname{pair}\left(a_{v_{j}}, b_{v_{j+1}}\right)$.

Proof. (1) Since the augmentation position cycles, the positions augment equally often. There are $t^{2}$ occasions of a position not augmenting, so each augments all but $t$ times. Since the amount by which each position increases is a multiple of $t$, after $t^{2}$ steps the walk returns to the original vector. To make $C_{t}$ a cycle, we need distinctness of the vertices.

(2) There are $t^{2}$ vectors of the specified form; we show that all arise. If $v_{j}$ has the specified form and the position of the first $q$ is congruent to $j$ modulo $t$ (which is true for $j=1$ ), then those statements remain true for $j+1$, by construction. Also the value of $q$ increases, except when $j \equiv t \bmod t$. Therefore, letting $i$ be the position of the first $q$, the pairs $(q, i)$ determining the vector cycle through $(1,1),(2,2) \ldots,(t, t)$, then $(t, 1),(1,2) \ldots,(t-1, t)$, then $(t-1,1),(t, 2), \ldots,(t-2, t)$, and so on until $(2,1),(3,2), \ldots,(1, t)$ (see the example of $C_{4}$ above). Thus $C_{t}$ visits all $t^{2}$ vectors of this form and is a cycle.

(3) The arrow from $v_{j}$ to $v_{j+1}$ represents the pair $\left(a_{v_{j}}, b_{v_{j+1}}\right)$. Since their subscripts agree in coordinate $i$, where $i \equiv j \bmod t$, they form an incomparable pair. 
Lemma 6.7 The arrows in $C_{t}$ represent a set of $t^{2}$ pairs that are 2 -conflicted in $\prod_{i=1}^{t} S_{t}$.

Proof. Consider distinct pairs $\left(a_{v_{i}}, b_{v_{i+1}}\right)$ and $\left(a_{v_{j-1}}, b_{v_{j}}\right)$; that is, with $j \neq i+1$. By Lemma 6.3, it suffices to that if $a_{v_{i}}$ and $b_{v_{j}}$ are incomparable, then $a_{v_{j-1}}<b_{v_{i+1}}$.

If $a_{v_{i}} \| b_{v_{j}}$, then $v_{i}$ and $v_{j}$ share some value $q$ in some position. The common value $q$ may be the "higher" (ending) value or the "lower" (beginning) value in each $t$-tuple.

If $q$ is the beginning value in $v_{i}$, then the values in $v_{i+1}$ are all at least $q+1$ (except that the first is $q$ when $i \equiv 1 \bmod t)$. The corresponding values in $v_{j-1}$ are different, since they are all at most $q$ (and the first is $q-1$ unless $j=i+1$ ). Hence $a_{v_{j-1}}<b_{v_{i+1}}$. The case where the shared value is the last in $v_{j}$ follows symmetrically.

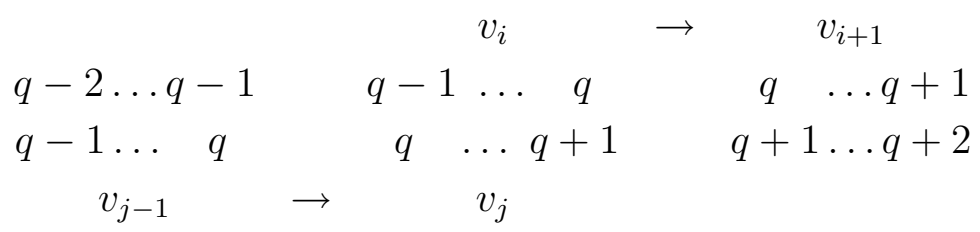

The remaining case is where $r$ is the position of the first $q$ in $v_{i}, s$ is the position of the last $q$ in $v_{j}$, and $1<r \leq s$, as shown above. Now $r$ is the position of the last $q$ in $v_{i+1}$, and $s$ is the position of the first $q$ in $v_{j-1}$. If $a_{v_{j-1}} \| b_{v_{i+1}}$ then $s \leq r$. Now $r=s$, and hence $j=i+1$.

Theorem 6.8 If $n_{1}, \ldots, n_{t}$ are all at least $t$, then $\operatorname{dim}_{2} \prod_{i=1}^{t} S_{n_{i}}=\sum_{i=1}^{t} n_{i}$.

Proof. For $t=2$, the claim was proved earlier, so assume $t \geq 3$.

By Lemma 6.7, the claim holds when $n_{i}=t$ for $1 \leq i \leq t$. When $\prod_{i=1}^{t} n_{i}>t^{t}$, begin with $C_{t}$ on the subgrid $[t]^{t}$. To this set of arrows, add the arrow from $u(i, j)$ to $u^{\prime}(i, j)$ for $1 \leq i \leq t$ and $t<j \leq n_{i}$, where $u(i, j)$ has $j$ in position $i$ and 1 everywhere else, while $u^{\prime}(i, j)$ has $j$ in position $i$ and 2 everywhere else. Since $u(i, j)$ and $u^{\prime}(i, j)$ agree in position $i$, the arrow represents an incomparable pair.

We claim that the resulting set is 2-conflicted. It is immediate that $u(i, j)$ and $u^{\prime}\left(i, j^{\prime}\right)$ differ in all coordinates when $j \neq j^{\prime}$, as do $u(i, j)$ and $u^{\prime}\left(i^{\prime}, j^{\prime}\right)$ when $i \neq i^{\prime}$, the latter since $t \geq 3$. Hence it suffices to compare $u(i, j)$ and $u^{\prime}(i, j)$ with $v_{p}$ and $v_{p+1}$ from $C_{t}$. If $v_{p}$ and $u^{\prime}(i, j)$ agree somewhere, then $v_{p}$ begins or ends with 2 . Now $v_{p+1}$ has no 1 and no $j$, so it agrees with $u(i, j)$ in no coordinate.

The same idea gives a weaker lower bound for larger $k$. We do not know how close it is to optimal. For $k \geq 4$, concatenating $k$-realizers of 2 -fold products of standard examples yields $\epsilon+\sum_{i=1}^{t}\left(n_{i}-1\right)$ as a trivial upper bound, where $\epsilon$ is 1 for odd $t$ and 0 for even $t$.

Theorem 6.9 If $n_{1}, \ldots, n_{t}$ are integers, all at least 2 , then $\operatorname{dim}_{k}\left(\prod_{i=1}^{t} S_{n_{i}}\right) \geq \sum_{i=1}^{t}\left(n_{i}-2\right)$. 
Proof. We produce a $k$-conflicted set of this size. Considering the grid $\prod_{i=1}^{t}[n]^{t}$, we form a set of $\sum_{i=1}^{t}\left(n_{i}-2\right)$ arrows. For $1 \leq i \leq t$ and $3 \leq j \leq n_{i}$, add the arrow from $u(i, j)$ to $u^{\prime}(i, j)$, where $u(i, j)$ has $j$ in position $i$ and 1 everywhere else, while $u^{\prime}(i, j)$ has $j$ in position $i$ and 2 everywhere else.

We observed in the proof of Theorem 6.8 that $\left(a_{u(i, j)}, b_{u^{\prime}(i, j)}\right)$ is an incomparable pair and that $a_{u(i, j)}<b_{u^{\prime}\left(i^{\prime}, j^{\prime}\right)}$ unless $i=i^{\prime}$ and $j=j^{\prime}$. That is, if $(x, y)$ and $\left(x^{\prime}, y^{\prime}\right)$ are two of these incomparable pairs, then $x<y^{\prime}$ and $x^{\prime}<y$. By Lemma 6.3, the set is $k$-conflicted.

\section{References}

[1] V. B. Alekseev, The number of monotone $k$-valued functions. (Russian) Problemy Kibernet. 28 (1974), 5-24, 278; correction, ibid. 29 (1974), 248.

[2] K. Baker, Dimension join-independence and breadth in partially ordered sets, manuscript.

[3] B. Dushnik and E.W. Miller, Partially ordered sets, Amer. J. Math. 63 (1941), 600-610.

[4] K. Engel, Optimal representations of partially ordered sets and a limit Sperner theorem. European J. Combin. 7 (1986), 287-302.

[5] K. Engel, Sperner Theory. Encyclopedia of Mathematics and its Applications, 65 (Cambridge University Press, 1997), 317-324.

[6] J. R. Griggs, J. Stahl, W. T. Trotter, A Sperner theorem on unrelated chains of subsets. J. Combin. Theory Ser. A 36 (1984), 124-127.

[7] B. V. Gnedenko and A. N. Kolomogorov, Limit Distributions for Sums of Independent Random Variables, (Addison-Wesley, 1968), 233.

[8] Ibid., 60 .

[9] T. Hiraguchi, On the dimension of partially ordered sets. Sci. Rep. Kanazawa Univ. 1 (1951), 77-94.

[10] T. Hiraguchi, On the dimension of orders, Sci. Rep. Kanazawa Univ. 4 (1955), 1-20.

[11] D. Kelly, On the dimension of partially ordered sets, Discrete Math. 35 (1981), 135-156.

[12] D. Kelly and W.T. Trotter, Jr. Dimension theory for ordered sets, in: I. Rival, ed. Ordered Sets (1982), 171-212 
[13] C. Lin, The dimension of the Cartesian product of posets. Discrete Math. 88 (1991), 79-92.

[14] O. Ore, Theory of graphs. Amer. Math. Soc. Colloq. Publ., Vol. XXXVIII (Amer. Math. Soc., Providence, R.I., 1962), x+270 pp.

[15] K. Reuter, On the dimension of the Cartesian product of orders and relations, Order 6 (1989), 277-293.

[16] W.T. Trotter, Jr., A generalization of Hiraguchi's inequality for posets. J. Comb. Theory Ser. A 20 (1976), 114-123.

[17] W.T. Trotter, Jr., The dimension of the Cartesian product of partial orders, Discrete Math. 52 (1985), 255-263. 